\title{
Exclusion rates in randomized controlled trials of treatments for physical conditions: a systematic review
}

\author{
Jinzhang $\mathrm{He}^{1}$, Daniel R. Morales ${ }^{2}$ and Bruce Guthrie ${ }^{3 *}$
}

\begin{abstract}
Background: The generalisability of randomized controlled trials (RCTs) can be uncertain because the impact of exclusion criteria is rarely quantified. The aim of this study was to systematically review studies examining the percentage of clinical populations with a physical health condition who would be excluded by RCTs of treatments for that condition.

Methods: Medline and Embase were searched from inception to Feb 11th 2018. Two reviewers independently completed screening, full-text review, data extraction and risk-of-bias assessment. The primary outcome was the percentage of patients in the clinical population who would have been excluded from each examined trial. Subgroup analyses examined exclusion by population setting, publication date and funding source.
\end{abstract}

Results: Titles/abstracts $(20,754)$ were screened, and 50 studies were included which reported exclusion rates from 305 trials of treatments in 31 physical conditions. Estimated rates of exclusion from trials varied from $0 \%$ to 100\%, and the median exclusion rate was $77.1 \%$ of patients (interquartile range $55.5 \%$ to $89.0 \%$ exclusion). Median exclusion rates for trials in common chronic conditions were high, including hypertension 83.0\%, type 2 diabetes $81.7 \%$, chronic obstructive pulmonary disease $84.3 \%$, and asthma $96.0 \%$. The most commonly applied exclusion criteria related to age, co-morbidity and co-prescribing, whereas more implicit criteria relating to life expectancy or functional status were not typically examined. There was no evidence that exclusion varied by the nature of the clinical population in which exclusion was evaluated or trial funding source. There was no statistically significant change in exclusion rates in more recent compared with older trials.

Conclusions: The majority of trials of treatments for physical conditions examined excluded the majority of patients with the condition being treated. Almost a quarter of the trials studied excluded over $90 \%$ of patients, more than half of trials excluded at least three quarters of patients, and four out of five trials excluded at least half of patients. A limitation is that most studies applied only a subset of eligibility criteria, so exclusion rates are likely under-estimated. Exclusion from trials of older people and people with co-morbidity and co-prescribing is increasingly untenable given population aging and increasing multimorbidity.

Trial registration: PROSPERO registration CRD42016042282.

Keywords: Randomized controlled trial [V03.175.250.500.500], External validity, Generalizability, Multimorbidity [N05.715.350.225.500], Aged [M01.060.116.100], Systematic review [V03.850], Real-world evidence

\footnotetext{
* Correspondence: bruce.guthrie@ed.ac.uk

${ }^{3}$ Centre for Population Health Sciences, University of Edinburgh, Doorway 3

Old Medical School, Teviot Place, Edinburgh EH8 9AG, Scotland

Full list of author information is available at the end of the article
}

(c) The Author(s). 2020 Open Access This article is distributed under the terms of the Creative Commons Attribution 4.0 International License (http://creativecommons.org/licenses/by/4.0/), which permits unrestricted use, distribution, and reproduction in any medium, provided you give appropriate credit to the original author(s) and the source, provide a link to the Creative Commons license, and indicate if changes were made. The Creative Commons Public Domain Dedication waiver (http://creativecommons.org/publicdomain/zero/1.0/) applies to the data made available in this article, unless otherwise stated. 


\section{Background}

Randomized controlled trials (RCTs) are the gold standard method for evaluating the efficacy of treatments because well-designed RCTs minimize bias and confounding. They therefore maximize internal validity, giving confidence that the results are true for the trial population studied. However, trial populations are often highly selected, which may weaken the generalizability of RCT evidence in the sense of leaving uncertainty that the results apply to everyone with the condition in clinical practice $[1,2]$. Some exclusions from RCTs are justifiable (e.g., where an individual is allergic to a medicine). However, Van Spall et al. estimated that $84.1 \%$ of trials published in high-impact general medical journals between 1994 and 2006 had poorly justified patient exclusion criteria [3].

A number of studies have shown that various landmark RCTs measuring treatment effects, many of which underpin guideline recommendations and influence regulatory decision-making, exclude large proportions of people with the condition being treated [4, 5]. Older people, women, and people with co-morbidity or coprescribing are noticeably excluded from trials [3, 6, 7]. Although there is some evidence that women and older people are better represented in newer trials, they remain under-represented compared with the wider population [7]. These patterns of exclusion do not represent the realities of current and future clinical practice. Most people with any chronic condition have co-morbidity, and multimorbidity is the norm in older people $[8,9]$. Therefore, guideline-recommended treatment in routine practice will often require significant extrapolation from RCT evidence [10, 11], where strict RCT eligibility criteria lead to trial populations significantly differing from clinical populations seen in routine practice $[12,13]$.

The problem that strict RCT eligibility criteria pose for generalizing from RCT-derived evidence is well known [14, 15]. However, the extent to which trials assessing treatment effects across different conditions exclude patients seen and treated in clinical practice is uncertain. The aims of this study were to undertake a systematic review of studies estimating the percentage of people with a chronic physical condition who would be excluded by RCTs of treatment for that condition and to examine how exclusion rates varied for different diseases, for different clinical populations, and over time.

\section{Methods}

\section{Search strategy}

A systematic review was undertaken searching the Medline and Embase databases from inception to 11 February 2018 for all studies comparing the percentage of people from a 'clinical' population with a physical condition who would have been excluded from one or more trials of treatment intended for that condition. The search strategy is detailed in Additional file 1.

\section{Inclusion criteria}

We included studies published that explicitly examined the percentage of people with a chronic physical condition in a defined clinical population who would have been eligible for one or more selected RCTs of an individual patient treatment for that condition (including medication, surgery and other non-pharmacological interventions). The clinical populations included were not restricted in terms of their setting or method of sampling and therefore could be any of unselected patients seen in clinical practice in primary or specialist care, patients in clinical or research registries, or research cohorts identified or recruited in these settings. However, the appropriateness of the clinical population used to examine exclusion from a particular trial was examined as part of risk-of-bias evaluation.

\section{Exclusion criteria}

We excluded studies examining eligibility for trials of mental health conditions, studies that were not published in English, studies that did not explicitly report the percentage of patients eligible for trials or where percentages of patients eligible could not be calculated from the available data (e.g., those comparing recruited with non-recruited patients without examining exclusion in an underlying clinical population), and studies examining eligibility for a hypothetical trial or applying a set of common exclusion criteria from multiple trials instead of using actual exclusion criteria from single trials. Since estimated exclusion rates in very small clinical populations are likely to be imprecise, we also excluded studies where eligibility was calculated in a clinical population that included fewer than 100 patients.

\section{Selection of studies}

All titles and abstracts were independently screened by two reviewers to identify papers for full-text review. Full-text review and data extraction were carried out independently by two reviewers on the basis of the published protocol [16], and disagreements were resolved by discussion to reach consensus.

\section{Data extraction and quality assessment}

Data extraction was carried out by a minimum of two reviewers, involving a third reviewer where necessary, and disagreements were resolved by discussion to reach consensus. Data extracted for each study included the condition of interest and a description of comparison clinical population, including the purpose of the clinical population dataset (e.g., clinical registry and electronic health record data), health-care setting and location, the 
date of clinical population recruitment or identification, clinical population size, and the diagnostic criteria used to define the clinical population. These data were used to make an assessment of bias on the overall appropriateness of the clinical population. Extracted data for the underlying trials examined by each study included the rationale for the choice of trials examined, the type of intervention or treatment in the trial, the listed trial eligibility criteria that were applied (or not) to each clinical population to estimate exclusion rate, and the trial's source of funding (pharmaceutical versus non-pharmaceutical).

The primary outcome extracted was the percentage of patients in the clinical population who would have been excluded for each trial examined and the reported 95\% confidence interval $(\mathrm{CI})$ of this percentage (which was calculated if not reported by the authors).

\section{Risk-of-bias assessment}

There is no published risk-of-bias tool to assess the kinds of studies examined. We therefore developed three pre-specified risk-of-bias criteria that were independently assessed by two reviewers, namely:

1) How the reviewed paper selected trials to examine. We evaluated whether there was a systematic approach to trial selection (e.g., systematic search of the literature) or a clearly stated justification for the choice of trials and whether that justification was judged to be adequate. Studies were considered to be at low risk of bias if selection rationale were clearly stated and judged to be justifiable; otherwise, they were considered to be at high risk of bias.

2) The appropriateness of each trial-clinical population pair. The appropriateness of each trialclinical population pair was assessed in relation to how well the clinical population appropriately represented the population for whom the treatment evaluated in the trial was intended or suitable. For example, a primary care population of people with heart failure is appropriate for a trial of betablockers or angiotensin-converting enzyme inhibitors used as long-term treatment [4], whereas an emergency department population is appropriate for a trial of treatment in acute, decompensated heart failure [17]. Studies were considered to be at low risk of bias if the clinical population was judged to be representative of real-world populations for which the trial treatment was intended or indicated, at high risk of bias if the clinical population was not considered to be representative of real-world populations for which the trial treatment was intended or indicated, and at unclear risk of bias if insufficient information was provided for assessment.
3) The choice of trial eligibility criteria to examine. The choice of trial eligibility criteria assessed in relation to the stated criteria applied and not applied. Studies were considered to be at low risk of bias for the choice of trial eligibility criteria assessed in relation to the stated criteria if they clearly stated that all important or common criteria were applied; otherwise, studies were considered to be at high risk of bias.

\section{Data synthesis and analysis}

Some trials were evaluated in more than one clinical population. In this situation, the trial-clinical population pair with the lowest percentage of patients was selected for analysis in order to obtain the most conservative estimate of the percentage of patients excluded. For the remaining trial-clinical population pairs, the overall median, range and interquartile range for the primary outcome (the estimated percentage of the clinical population excluded by each trial) were calculated and repeated for condition groups (cardiovascular conditions, diabetes, respiratory conditions, cancer, rheumatoid arthritis (RA), human immunodeficiency virus (HIV) and other conditions) and for individual conditions. Variation was further examined by using linear regression to model unadjusted and adjusted differences in the percentage excluded by each trial in relation to whether the clinical population was recruited from primary or specialist care, whether the trial examined was publicly funded or industry-funded, the date of trial publication (with trials grouped into quartiles of publication date with equal numbers of trials in each group: 19941999, 2000-2003, 2004-2011 and 2012-2018), and riskof-bias assessment (low risk of bias versus high or unclear risk of bias).

\section{Results \\ Study characteristics}

The searches identified 21,885 articles with a further 18 identified from other sources, including examination of references of included studies. Non-duplicate documents $(20,754)$ were screened, and 222 full-text articles were examined. Fifty studies that examined trial eligibility in 57 distinct clinical populations were included (Fig. 1). Twenty of the reference clinical populations examined were primary care or community samples: seven derived from electronic clinical datasets, three clinical registries, five research registries, and five survey-derived populations. Thirty-seven of the clinical populations examined were specialist samples: 19 derived from record review of various kinds, four clinical registries, and 14 research registries. Characteristics of all 50 included studies and all 57 reference clinical populations are shown in supplementary table S1. The 50 studies provided data on the 


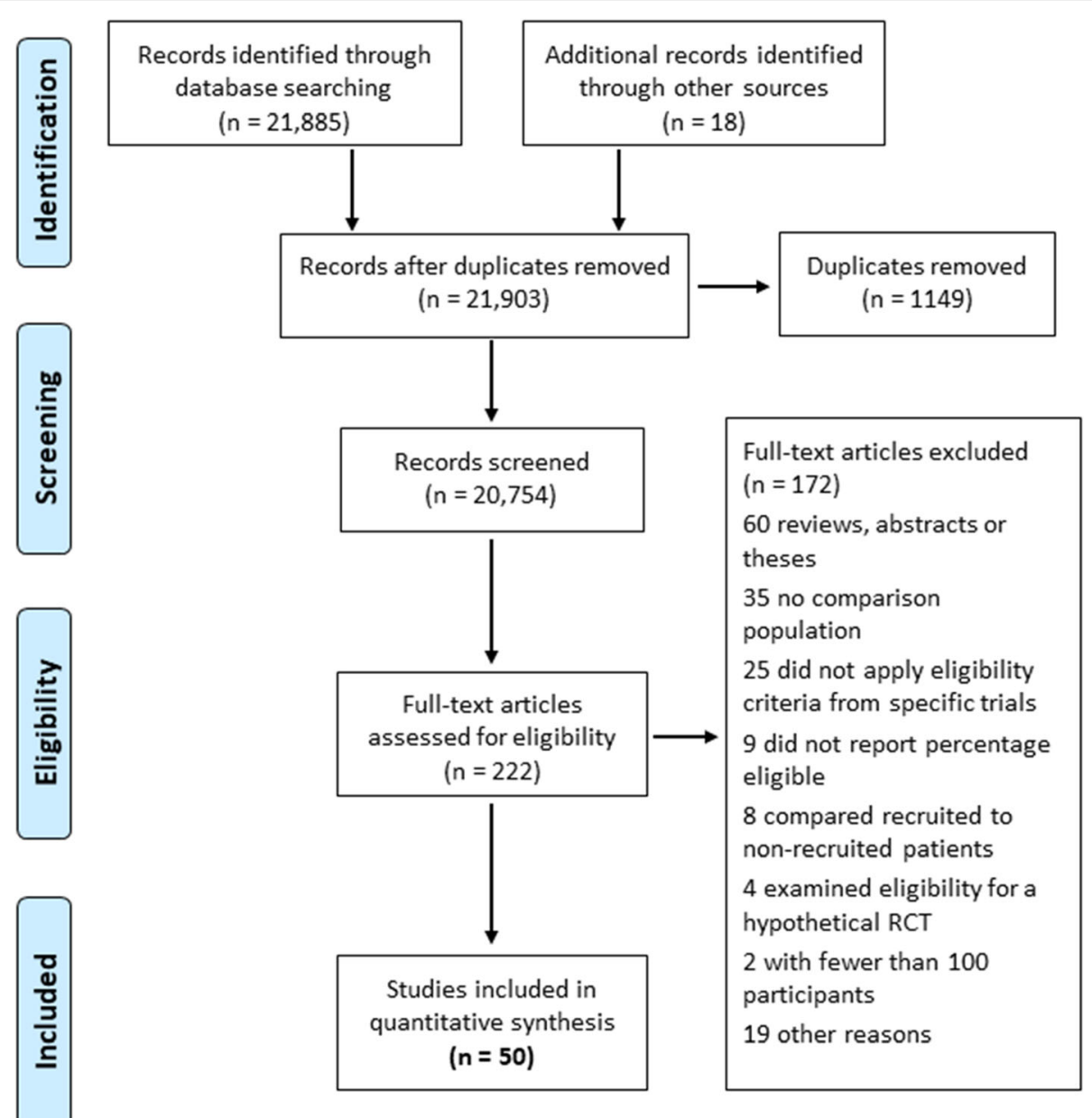

Fig. 1 Flow diagram of identification, screening and eligibility assessment

proportion of the reference clinical population that would have been excluded by 305 trials. Characteristics of all the trials examined by these studies are shown in supplementary tables S2, S3, S4, S5, S6, S7, S8, S9, S10, S11, S12, S13, S14, S15 and S16. Eighty-one (26.6\%) of the trials examined by the 50 studies were published in 1994-1999, $78(25.6 \%)$ in $2000-2003,75(24.6 \%)$ in 2004-2011, and 67 (22.0\%) in 2012-2018. Seventy-five (24.6\%) trials were publicly funded, 203 (66.6\%) industry-funded, and 27 (8.9\%) did not record their source of funding. Included studies examined trial exclusion in 31 physical conditions (seven cardiovascular, three diabetes, three respiratory, eight types of cancer, RA, HIV infection, and eight other conditions) (Table 1), and there was considerable heterogeneity in the treatments being trialed (supplementary table S2). The trials examined were most frequently evaluating treatments for RA (51 trials; 16.7\%), chronic obstructive pulmonary disease (COPD) (51 trials; 16.7\%), HIV infection (31 trials; $10.2 \%$ ), heart failure (25 trials; $8.2 \%$ ) and hypertension (22 trials; $7.2 \%)$.
Percentage of the clinical population excluded from trials Across all 305 trials, the median rate of exclusion was $77.1 \%$ (range $0-100 \%$ ) of patients, varying from a median of $42.0 \%$ for HIV trials to a median of $89.4 \%$ for respiratory trials (Table 1, Fig. 2). Only 16 (5.2\%) trials excluded less than $25 \%$ of patients, whereas 159 (52.1\%) excluded at least $75 \%$. At single-condition level, trials of treatments in atrial fibrillation excluded the fewest patients (median 34.9\%, range 32.3-41.2\%) and trials of treatments in asthma the most (median $96.0 \%$, range $64.0-100 \%$ ). Notably, exclusion rates for the most common chronic conditions were high, including hypertension $83.0 \%$, lipid-lowering drugs in primary prevention $85.9 \%$, type 2 diabetes $81.7 \%$, COPD $84.3 \%$ and asthma $96.0 \%$.

\section{Inclusion and exclusion criteria used by studies to estimate exclusion rates}

It was only explicit which eligibility criteria had been used to determine exclusion rates in the clinical population for $174(57.4 \%)$ of trials. The most commonly 
Table 1 Percentage of the clinical population excluded by condition studied

\begin{tabular}{|c|c|c|}
\hline & Number of trials & Median percentage excluded (range excluded) \\
\hline All conditions & 305 & 77.1 (0.0 to 100.0) \\
\hline Cardiovascular conditions & 81 & 74.7 (1.6 to 98.8$)$ \\
\hline Heart failure & 25 & $65.0(18.8$ to 92.0$)$ \\
\hline Hypertension & 22 & $83.0(1.6$ to 98.8$)$ \\
\hline Stroke/transient ischemic attack & 21 & 83.6 (33.2 to 98.4$)$ \\
\hline Atrial fibrillation & 4 & 34.9 (32.3 to 41.2$)$ \\
\hline Coronary heart disease & 4 & 53.1 (2.8 to 84.5$)$ \\
\hline Lipid lowering for primary prevention & 4 & 85.9 (69.7 to 89.1$)$ \\
\hline Secondary prevention of myocardial infarction & 1 & 76.8 \\
\hline Diabetes mellitus & 16 & 88.1 (29.8 to 99.0$)$ \\
\hline Type 2 diabetes & 7 & 81.7 (49.3 to 96.5$)$ \\
\hline Diabetic ulcers & 7 & 93.3 (29.8 to 99.0$)$ \\
\hline Type 1 diabetes & 2 & 91.6 (87.5 to 95.6$)$ \\
\hline Respiratory conditions & 78 & 89.4 (42.4 to 100.0) \\
\hline COPD & 51 & 84.3 (42.4 to 100.0) \\
\hline Asthma & 17 & 96.0 (64.0 to 100.0$)$ \\
\hline Bronchiectasis & 10 & 80.1 (49.0 to 93.0) \\
\hline Cancer & 24 & 56.6 (13.6 to 81.2) \\
\hline Breast cancer & 12 & 56.6 (28.9 to 81.2 ) \\
\hline Lung cancer & 3 & 71.4 (65.4 to 71.9$)$ \\
\hline Renal cancer & 3 & $13.6(13.6$ to 48.5$)$ \\
\hline Colorectal cancer & 2 & 66.7 (65.7 to 67.6) \\
\hline Bladder cancer & 1 & 45.3 \\
\hline Stomach cancer & 1 & 41.3 \\
\hline Lymphoma & 1 & 70.4 \\
\hline Prostate cancer & 1 & 57.1 \\
\hline Rheumatoid arthritis & 51 & 84.0 (56.0 to 98.7$)$ \\
\hline HIV infection & 32 & 42.0 (0.0 to 67.6$)$ \\
\hline Other conditions & 23 & 58.3 (23.7 to 88.9$)$ \\
\hline Venous thromboembolism prophylaxis & 9 & 41.5 (23.7 to 78.8$)$ \\
\hline Venous ulcers & 7 & 83.6 (58.3 to 88.9$)$ \\
\hline Brain injury & 2 & 40.5 (35.9 to 45.0$)$ \\
\hline Pressure ulcers & 1 & 34.7 \\
\hline Alzheimer's disease & 1 & 86.5 \\
\hline Fibromyalgia & 1 & 52.1 \\
\hline Irritable bowel syndrome & 1 & 73.1 \\
\hline Incisional hernia & 1 & 62.5 \\
\hline
\end{tabular}

Abbreviations: COPD chronic obstructive pulmonary disease, HIV human immunodeficiency virus

a Where there is only one trial-clinical population comparison, the number reported is the value for that comparison; where there are two, the median reported is the midpoint value between the two

reported eligibility criterion used to determine exclusion rates was disease severity for 142 trials $(81.4 \%$ of trials where this was reported), most commonly selecting patients with more severe or less well-controlled disease. Co-morbidity was reported as being used to determine exclusion rates for 119 (68.4\%) trials, usually as an exclusion criterion (117 [67.2\%] trials) but sometimes as an inclusion criterion (14 [8.0\%] trials, for example, to select patients at higher risk of cardiovascular disease in diabetes and atrial fibrillation trials). Age was reported 


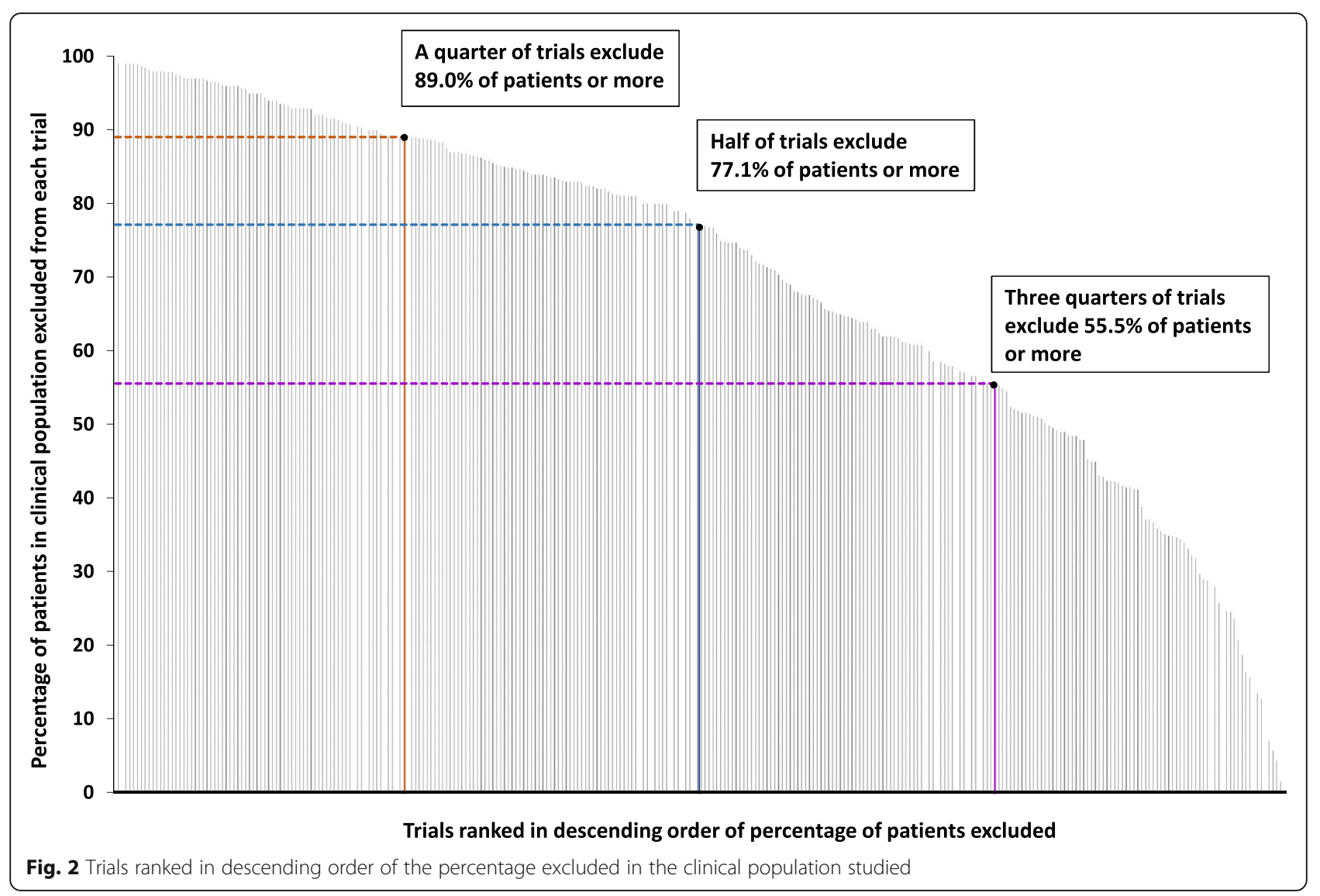

as used to determine exclusion rates in the clinical population for $86(49.4 \%)$ trials, most commonly using an upper age limit for eligibility and other criteria such as limited life expectancy and inability to comply with treatment for $56(32.2 \%)$ trials.

\section{Variation by type of condition}

HIV trials excluded the lowest percentage of patients amongst the different conditions (mean 38.4\%, 95\% CI 31.4 to 45.5 ) (Table 2). In unadjusted analysis, there were statistically significantly higher rates of exclusion for all other conditions compared with HIV trials, and cancer trials excluded $15.6 \%$ more patients, cardiovascular trials $31.8 \%$ more, respiratory trials $36.6 \%$ more, RA trials $44.6 \%$ more, and diabetes trials $42.4 \%$ more. When adjusted for all other variables, the results remained similar with significant differences in the percentage of patients excluded in trials of different conditions compared with HIV trials; cancer trials excluded $20.4 \%$ (95\% CI 8.8 to 48.4 ) more patients, cardiovascular trials $34.0 \%$ (95\% CI 24.0 to 44.0 ) more, respiratory trials $43.1 \%$ (95\% CI 31.9 to 54.4 ) more, RA trials $43.9 \%$ (95\% CI 33.4 to 54.4 ) more, and diabetes trials $46.8 \%$ (95\% CI 31.1 to 62.6$)$.
Variation by funding source, clinical population and trial publication date

Publicly funded trials excluded a mean of $58.2 \%$ of patients (95\% CI 52.3 to 64.1), and industry-funded trials excluded $15.7 \%$ more (95\% CI 9.6 to 21.7 ) in unadjusted analysis, but there was no statistically significant difference observed after adjustment (difference $-4.7 \%$, 95\% CI -11.0 to 1.6). Studies where the clinical population was recruited in primary care excluded a mean of $72.2 \%$ (95\% CI 69.0 to 75.5). In unadjusted analysis, studies where the clinical population was in specialist care excluded $6.2 \%$ (95\% CI -11.7 to -0.6$)$ more patients, but there was no statistically significant difference after adjustment (difference-3.0\%, 95\% CI -9.0 to 3.0). Trials published during 1994-1999 excluded $71.0 \%$ of patients (95\% CI 65.6 to 76.5 ) on average. This was no different in later time periods in unadjusted analysis. Although estimated differences were larger in adjusted analysis with fewer people excluded more recently, differences remained non-significant (2012-2018 difference compared with 1194-1999 -6.5\% (95\% CI -13.8 to $0.7, P=0.08$ ).

\section{Risk of bias}

In risk-of-bias assessment, 126 (41.3\%) of estimates of trial exclusion rates were assessed as low risk of bias, 104 
Table 2 Exclusion rates by trial characteristics

\begin{tabular}{|c|c|c|c|c|}
\hline Variable (number of trials) & Unadjusted coefficient $(95 \% \mathrm{Cl})^{\mathrm{a}}$ & $P$ value & Adjusted coefficient $(95 \% \mathrm{Cl})^{\mathrm{a}}$ & $P$ value \\
\hline \multicolumn{5}{|l|}{ Condition } \\
\hline HIV infection $(n=32)$ & Reference & & Reference & \\
\hline Cancer $(n=24)$ & $15.6(5.0$ to 26.2$)$ & $<0.001$ & 20.4 (8.8 to 32.0$)$ & $<0.001$ \\
\hline Cardiovascular $(n=81)$ & 31.8 (23.8 to 39.7$)$ & 0.003 & $34.0(24.0$ to 44.0$)$ & $<0.001$ \\
\hline Respiratory $(n=78)$ & 36.6 (27.8 to 45.3$)$ & $<0.001$ & 43.1 (31.9 to 54.2 ) & $<0.001$ \\
\hline Rheumatoid arthritis $(n=51)$ & 44.6 (36.9 to 52.2$)$ & $<0.001$ & 43.9 (33.4 to 54.4$)$ & $<0.001$ \\
\hline Diabetes $(n=16)$ & 42.4 (28.2 to 56.7$)$ & $<0.001$ & 46.8 (31.1 to 62.6$)$ & $<0.001$ \\
\hline Other conditions ( $n=23$ ) & 19.5 (9.2 to 29.8$)$ & $<0.001$ & $25.0(12.2$ to 37.8$)$ & $<0.001$ \\
\hline \multicolumn{5}{|l|}{ Trial funding source ${ }^{b}$} \\
\hline Public $(n=75)$ & Reference & & Reference & \\
\hline Industry $(n=203)$ & 15.7 (9.6 to 21.7$)$ & $<0.001$ & $-4.7(-11.0$ to 1.6$)$ & 0.1 \\
\hline \multicolumn{5}{|c|}{ Comparison clinical population setting } \\
\hline Primary care $(n=198)$ & Reference & & Reference & \\
\hline Specialist care $(n=107)$ & $-6.2(-11.7$ to -0.6$)$ & 0.03 & $-3.0(-9.0$ to 3.0$)$ & 0.3 \\
\hline \multicolumn{5}{|l|}{ Year of trial publication } \\
\hline 1994-1999 $(n=81)$ & Reference & & Reference & \\
\hline $2000-2003(n=78)$ & $-4.0(-11.4$ to 3.3$)$ & 0.28 & $-4.7(-10.8$ to 1.4$)$ & 0.1 \\
\hline $2004-2011(n=75)$ & -1.1 (-8.6 to 6.3$)$ & 0.76 & $-6.2(-13.1$ to 0.7$)$ & 0.08 \\
\hline $2012-2018(n=71)$ & $-0.3(-7.4$ to 7.9$)$ & 0.95 & $-6.5(-13.8$ to 0.7$)$ & 0.08 \\
\hline \multicolumn{5}{|l|}{ Risk of bias } \\
\hline $\operatorname{Low}(n=126)$ & Reference & & Reference & \\
\hline High/Unclear $(n=179)$ & 17.2 (12.2 to 22.2$)$ & $<0.001$ & $9.2(3.5$ to 14.8$)$ & 0.002 \\
\hline
\end{tabular}

(34.1\%) as high risk, and $75(24.6 \%)$ as unclear. High risk of bias was driven largely by the clinical population used in the comparison being judged as less appropriate for the treatment being trialed (supplementary tables S17 and S18). Comparisons with a low risk of bias had significantly lower exclusion rates (Table 2). Low-risk studies excluded $59.9 \%$ (95\% CI 55.7 to 64.1 ) of patients on average, and studies rated high/unclear risk of bias excluded 17.2\% more patients (95\% CI 12.2 to 22.2). After adjustment for other characteristics, studies rated high/unclear risk of bias excluded $9.2 \%$ more patients ( $95 \%$ CI 3.5 to 14.8 ).

\section{Trials where exclusion rates were estimated in multiple clinical populations}

Thirty-eight trials were examined in two or more clinical populations (Table 3), and 30 were trials of treatment for RA. Exclusion rates of nine RA trials were each estimated in three clinical populations [18], whereas exclusion rates for the remaining 21 were estimated in two clinical populations [19]. For the nine trials examined in three clinical populations, estimated exclusion rates were higher in every comparison in the Veterans' Affairs Rheumatoid Arthritis (VARA) cohort (median 97.4\%, range 75.6 to $98.4 \%$ ) when compared with the Rheumatoid Arthritis Investigators' Network (RAIN) database (median 89.6\%, range 74.7 to $91.6 \%$ ) and the National Register for Biologic Treatment cohort (median $80.0 \%$, range 56.0 to $92.4 \%$ ). In the remaining 21 trials, estimated exclusion rates in every comparison were higher in VARA (median 97.4\%, range 72.7 to $99.1 \%$ ) than in RAIN (median 89.0\%, range 64.9 to $93.5 \%$ ). Such differences would be expected given variation in the data collected by different registries and in the clinical population included (VARA, for example, is made up predominately of male veterans whereas RAIN is a less selected population of patients attending rheumatology clinics) [20]. Differences were more variable and sometimes larger for trials of treatments for the other conditions (atrial fibrillation, heart failure, acute myocardial infarction and COPD) examined in more than one clinical population, although there was no consistent pattern to explain this in relation to risk of bias or the nature of the clinical population (Table 3).

\section{Discussion}

\section{Summary of evidence}

This study examined estimated exclusion rates in clinical populations in 305 trials of treatments for physical 
Table 3 Consistency of findings when the same trial is examined in more than one clinical population

\begin{tabular}{|c|c|c|c|}
\hline Condition and trial & Clinical population & Overall risk of bias & $\begin{array}{l}\text { Percentage excluded } \\
\text { Median percentage (range } \\
\text { for Aaltonen and Vashisht) }\end{array}$ \\
\hline \multirow{5}{*}{$\begin{array}{l}\text { Atrial fibrillation } \\
\text { ARISTOTLE (2011) }\end{array}$} & Yoon (record review for consenting patients in a single hospital) & High & 32.3 \\
\hline & Lee (primary care electronic medical record population data) & Low & 38.7 \\
\hline & Fanning (record review within multiple hospitals) & High & 39.5 \\
\hline & Desmaele (clinical registry in a single hospital) & High & 54.5 \\
\hline & Hagg (primary care electronic medical record population data) & Low & 71.1 \\
\hline \multirow{4}{*}{$\begin{array}{l}\text { Atrial fibrillation } \\
\text { ROCKET-AF (2011) }\end{array}$} & Yoon (record review for consenting patients in a single hospital) ${ }^{a}$ & High & 34.5 \\
\hline & Lee (primary care electronic medical record population data) & Low & 52.5 \\
\hline & Desmaele (clinical registry in a single hospital) & High & 60.7 \\
\hline & Fanning (record review within multiple hospitals) & High & 64.2 \\
\hline \multirow{4}{*}{$\begin{array}{l}\text { Atrial fibrillation } \\
\text { RE-LY (2009) }\end{array}$} & Yoon (record review for consenting patients in a single hospital) & High & 35.2 \\
\hline & Lee (primary care electronic medical record population data) & Low & 36.2 \\
\hline & Fanning (record review within multiple hospitals) & High & 47.4 \\
\hline & Desmaele (clinical registry in a single hospital) & High & 52.4 \\
\hline \multirow{3}{*}{$\begin{array}{l}\text { Heart failure } \\
\text { MERIT-HF (2000) }\end{array}$} & Constantino (record review in a single hospital) ${ }^{a}$ & Unclear & 48.0 \\
\hline & Jost (clinical registry in a single hospital) & Low & 58.8 \\
\hline & Masoudi (National Heart Failure Project registry) & High & 82.6 \\
\hline \multirow{2}{*}{$\begin{array}{l}\text { Heart failure } \\
\text { RALES (1999) }\end{array}$} & Masoudi (National Heart Failure Project registry) ${ }^{a}$ & High & 74.7 \\
\hline & Costantino (record review in a single hospital) & Unclear & 76.0 \\
\hline \multirow{2}{*}{$\begin{array}{l}\text { Acute myocardial infarction } \\
\text { GUSTO (1993) }\end{array}$} & Krumholz (National Research Registry of Myocardial Infarction) ${ }^{a}$ & High & 84.5 \\
\hline & Krumholz (Cooperative Cardiovascular Project registry) & High & 90.6 \\
\hline \multirow{2}{*}{$\begin{array}{l}\text { COPD } \\
\text { POET-COPD (2011) }\end{array}$} & Kruis (seven primary care databases) ${ }^{a}$ & High & 77.0 \\
\hline & Halpin (primary care research database) & Low & 88.2 \\
\hline \multirow{2}{*}{$\begin{array}{l}\text { COPD } \\
\text { UPLIFT (2009) }\end{array}$} & Kruis (seven primary care databases) ${ }^{a}$ & High & 58.0 \\
\hline & Halpin (primary care research database) & Low & 77.5 \\
\hline \multirow{3}{*}{$\begin{array}{l}\text { Rheumatoid Arthritis } \\
9 \text { trials }^{\mathrm{b}}\end{array}$} & Aaltonen (National Register for Biologic Treatment) ${ }^{a}$ & High & $80.0(56.0$ to 92.4$)$ \\
\hline & Vashisht (RA Investigators' Network database research registry) & High & 89.6 (74.7 to 91.6$)$ \\
\hline & Vashisht (Veterans' Affairs Rheumatoid Arthritis research registry) & Unclear & $97.4(75.6$ to 98.4$)$ \\
\hline \multirow{2}{*}{$\begin{array}{l}\text { Rheumatoid Arthritis } \\
21 \text { trials }^{\mathrm{b}}\end{array}$} & Vashisht (RA Investigators' Network database research registry) $)^{a}$ & Unclear & $89.0(64.9$ to 93.5$)$ \\
\hline & Vashisht (Veterans' Affairs Rheumatoid Arthritis research registry) & High & 97.4 (72.7 to 99.1$)$ \\
\hline
\end{tabular}

${ }^{a}$ Marked trials with most conservative estimate of percentage of patients excluded were analysed

${ }^{b}$ See supplementary table S14 for individual trial comparisons

conditions. Almost a quarter of the trials studied excluded $90 \%$ or more of patients, more than half of trials excluded more than $75 \%$ of patients, and four out of five trials excluded more than $50 \%$ of patients. There was variation in exclusion depending on the condition studied, but exclusion rates did not differ between studies using primary versus specialist care clinical populations to evaluate exclusion rates or between trials that were publicly versus industry-funded. There was no strong evidence that rates of exclusion had changed over time. A third of studies were at high risk of bias, most commonly because the clinical population used was not appropriate for the trial examined, and a further quarter of studies were at unclear risk of bias. Exclusion rates were lower for studies at low risk of bias where median exclusion was $60.8 \%$, although two thirds of low risk-of-bias studies would still have excluded more than $50 \%$ and one third more than $75 \%$ of patients.

\section{Strengths and limitations}

A strength of the study is the systematic approach to identify and examine the underlying literature by using a deliberately broad search strategy to maximize sensitivity. However, the nature of the literature examined and the fact that there are no clear reporting criteria for such studies make it possible that some studies were not identified. Despite this, estimated exclusion rates in 305 trials in 57 clinical populations were included. A key 
observation is that examined studies were heterogeneous in a variety of ways. Underlying studies varied in how they selected trials to compare, in their choice of clinical population, and in the trial inclusion and exclusion criteria they applied. Some of the observed variation in exclusion rates likely reflects the choices made, but these were not always explicit in the included studies. This may be related to the fact that there are no clear criteria for the conduct of such studies. A further limitation is that we excluded comparisons with fewer than $100 \mathrm{pa}$ tients in order to avoid imprecise estimates for common conditions (although, in practice, only two studies were excluded as a result). Finally, most of the underlying studies applied only a subset of eligibility criteria, most commonly age, co-morbidity and co-prescribing because these are easily applied to the data contained in coded data extracted from electronic health records and clinical or research registries. The implication is that true exclusion rates are likely even higher than reported here because of unexamined explicit criteria and because trial recruitment also involves the application of implicit criteria by researchers (such as the presence of frailty and whether an individual is perceived to be likely to adhere to trial procedures).

\section{Comparison with other literature}

Exclusion and inclusion criteria are not always clearly reported in trial publications. For example, $56 \%$ of 255 cancer RCTs published in leading journals had discrepancies between eligibility criteria listed in protocols and those listed in the papers reporting results, and $96.7 \%$ of these discrepancies imply that the trial population was broader than it actually was [21]. Examining RCTs published in high-impact journals 1994-2006, Van Spall et al. found co-morbidity, age and co-prescribing used as exclusion criteria in the majority of the 283 trials examined, usually without any explicit justification [3]. A study of 4341 RCTs published in four high-impact general medical journals found that $29 \%$ had upper age limits for inclusion that were rarely explicitly justified. Although the percentage of trials with upper age limits declined somewhat between 1998 and 2015, absolute change over time was small [22], and only 7\% of RCTs published in 2012 were specifically conducted in older patients [23]. Of 319 ongoing RCTs for 10 common conditions registered with ClinicalTrials.gov in 2014, 79\% excluded patients with common co-morbidities [24]. Studies of trials in individual conditions have similar findings. Only one of 112 RCTs of secondary prevention of cardiovascular disease published in 2010-2012 justified the exclusion criteria applied [25]. Two thirds of RCTs for type 2 diabetes had upper age limits for inclusion, three quarters excluded a range of co-morbidities, and only $1.4 \%$ of the 440 RCTs examined were specifically in older adults [26]. However, this literature does not quantify the impact of inclusion and exclusion criteria on eligibility as we have done here.

\section{Implications for policy, practice and research}

Exclusion of patients from trials matters only if the exclusion criteria are effect modifiers of treatment [27], meaning that the benefits or harms of treatment (or both) systematically vary in the included versus the excluded. This review found that trial evidence is typically derived from narrow populations which are usually selected to have higher risk of outcomes expected to be improved by treatment (e.g., by selective inclusion of patients at high cardiovascular risk) and usually selected to have lower risk of adverse effects (e.g., by selective exclusion of patients with co-morbidity, co-prescribing and frailty).

Guideline developers, medicine regulators and clinicians therefore all face the problem of having to extrapolate RCT findings to excluded clinical populations where benefits and harms may be plausibly different. Simple extrapolation requires making assumptions that the benefits and harms of treatment are similar in included and excluded populations [28]. This is often reasonable but such assumptions do not always hold true. For example, trial-derived estimated numbers needed to treat (NNTs) for the use of angiotensin-converting enzyme inhibitors over about 3 years to prevent end-stage renal disease (ESRD) in chronic kidney disease are 9-25. Estimated NNTs to prevent ESRD in clinical populations are more than 100 because of lower baseline risk of ESRD and higher risk of competing mortality than observed in trial populations [29]. Adverse effects and harms from treatment are also usually higher in people with frailty and polypharmacy [30] and increase with age. Aspirin used after a cerebrovascular event in patients over 75 years old, for example, is associated with a fivefold increase in fatal bleeding compared with younger patients [31]. So even if treatment benefits are similar in trial and clinical populations, overall net benefit may still vary.

Careful attention to internal validity has improved the quality of trial evidence and its systematic synthesis, but generalizability and applicability are usually less explicitly considered [32]. Despite recommendations that systematic reviews should always discuss applicability of evidence [33], only a minority actually do [34]. There remains a clear place for efficacy trials in highly selected populations, but choosing to design such a trial is also effectively a declaration that the trialists have concerns that net benefit may be different in excluded populations. While more restrictive eligibility criteria for earlystage clinical trials may be appropriate when little is known about a treatment's safety and efficacy, enrolment of more diverse populations for later studies (or adaptive enrolment to include broader populations depending on 
initial efficacy findings) will help ensure a better understanding of the treatment's effect for all patients likely to benefit. In this regard, the US Food and Drug Administration is exploring recommendations around modernizing eligibility criteria for cancer clinical trials [35]. Furthermore, robust methods aimed at generating realworld evidence may help augment evidence from trials.

To facilitate judgements about applicability by clinicians, systematic reviewers, guideline developers and medicine regulators, journals and registries should require trialists to explicitly report and justify inclusion and exclusion criteria, should report data on who was excluded at screening (although much exclusion happens before formal eligibility screening), and ideally should report how the trial population compares with the clinical population from which it was recruited. Age, co-morbidity and co-prescribing exclusions in particular require justification, not least because aging populations mean that for most conditions older people with multimorbidity and polypharmacy will be an increasing percentage of the clinically treated population $[8,36]$.

Assessment of the applicability of evidence should be explicitly reported by systematic reviews and in guideline development. Extrapolation of evidence is inevitable but should be explicitly justified when recommendations are made for all patients with a condition based on trial evidence from narrow subsets of the clinical population. Alternatively, guideline developers may consider making more nuanced or stratified recommendations that account for differences between trial and clinical populations [28, 37]. Guideline development therefore needs to be more informed by evidence about applicability by making greater use of epidemiological data describing how the clinical population differs from trial populations. This is also relevant for medicine regulation, where a better understanding of differences between trial and real-world populations may help in risk-minimization planning, including in the design of post-authorization safety studies.

Finally, although this review found a large volume of evidence about exclusion, the quality of that evidence was variable. Future studies in this field should clearly justify their selection of trials to examine and prioritize landmark trials or those cited in high-quality guidelines since these most clearly define standards of practice. The clinical population used to examine eligibility should be clearly described, and its appropriateness for measuring exclusion rates in the trial being examined justified. Studies of exclusion should report all eligibility criteria applied and all criteria not applied and discuss the implications of this for interpreting the findings.

\section{Conclusions}

Most people with any of the physical conditions studied would be excluded from most trials of treatments for that condition. This is most commonly because the trial excludes older people and those with significant comorbidity or co-prescribing. Population aging, increasing multimorbidity and increasing polypharmacy make it imperative that evidence of treatment effectiveness better match the people whom we actually treat in clinical practice.

\section{Supplementary information}

Supplementary information accompanies this paper at https://doi.org/10. 1186/s13063-020-4139-0.

Additional file 1. Search strategy and Supplementary Tables.

\begin{abstract}
Abbreviations
$\mathrm{Cl}$ : Confidence interval; COPD: Chronic obstructive pulmonary disease; ESRD: End-stage renal disease; HIV: Human immunodeficiency virus; NNT: Number needed to treat; RA: Rheumatoid arthritis; RAIN: Rheumatoid Arthritis Investigators' Network; RCT: Randomized controlled trial; VARA: Veterans' Affairs Rheumatoid Arthritis
\end{abstract}

\section{Acknowledgements}

Not applicable.

\section{Authors' contributions}

BG and DM had the idea for the study, and designed it with $\mathrm{JH}$. JH led the conduct of the study. BG and DM contributed to searching, screening, review, analysis and interpretation. JH wrote the first draft, and all authors revised the draft and approved the submitted version. BG is the corresponding author, confirms that he had full access to all the data in the study, and takes responsibility for the integrity of the data and the accuracy of the data analysis. All authors are responsible for accuracy of the content.

\section{Funding}

The study design drew on work carried out with National Institute for Health Research (NIHR) Health Services and Delivery Research Programme grant NIHR HS\&DR 11/2003/27. The views expressed are those of the authors and not necessarily those of the NIHR or the Department of Health and Social Care. JH was funded by a vacation studentship from Medical Research Scotland (Vac-965-2016). The funders had no role in study design, analysis or the decision to publish. The review protocol was registered with PROSPERO (CRD42016042282) [16].

\section{Availability of data and materials}

Data sharing is not applicable to this article as no datasets were generated or analysed during the current study. All underlying studies and trials are documented and referenced in the supplementary file.

\section{Ethics approval and consent to participate}

Since the study was wholly literature based, ethical approval was not required.

\section{Consent for publication}

Not applicable.

\section{Competing interests}

The authors declare that they have no competing interests.

\section{Author details}

${ }^{1}$ Ninewells Hospital and Medical School, University of Dundee, James Arrott Drive, Dundee DD2 1SY, Scotland. ${ }^{2}$ Population Health and Genomics Division, University of Dundee, Mackenzie Building, Kirsty Semple Way, Dundee DD2 4BF, Scotland. ${ }^{3}$ Centre for Population Health Sciences, University of Edinburgh, Doorway 3 Old Medical School, Teviot Place, Edinburgh EH8 9AG, Scotland. 
Received: 19 July 2019 Accepted: 4 February 2020

Published online: 26 February 2020

\section{References}

1. Rothwell P. Factors that can affect the external validity of randomised controlled trials. PLoS Clin Trials. 2006;1(1):e9.

2. Rothwell PM. External validity of randomised controlled trials: to whom do the results of this trial apply? Lancet. 2005;365(9453):82-93.

3. Van Spall HGC, Toren A, Kiss A, Fowler RA. Eligibility criteria of randomized controlled trials published in high-impact general medical journals: a systematic sampling review. JAMA. 2007;297(11):1233-40.

4. Masoudi FA, Havranek EP, Wolfe P, Gross CP, Rathore SS, Steiner JF, et al. Most hospitalized older persons do not meet the enrollment criteria for clinical trials in heart failure. Am Heart J. 2003;146(2):250-7.

5. Saunders C, Byrne CD, Guthrie B, Lindsay RS, McKnight JA, Philip S, et al. External validity of randomized controlled trials of glycaemic control and vascular disease: how representative are participants? Diabet Med. 2013: 30(3):300-8.

6. Townsley CA, Selby R, Siu LL. Systematic review of barriers to the recruitment of older patients with cancer onto clinical trials. J Clin Oncol. 2005;23(13):3112-24

7. Lee PY, Alexander KP, Hammill BG, Pasquali SK, Peterson ED. Representation of elderly persons and women in published randomized trials of acute coronary syndromes. JAMA. 2001;286(6):708-13.

8. Barnett K, Mercer SW, Norbury M, Watt G, Wyke S, Guthrie B. Epidemiology of multimorbidity and implications for health care, research, and medical education: a cross-sectional study. Lancet. 2012;380:37-43.

9. Marengoni A, Angleman S, Melis R, Mangialasche F, Karp A, Garmen A, et al. Aging with multimorbidity: a systematic review of the literature. Ageing Res Rev. 2011;10(4):430-9.

10. Boyd CM, Darer J, Boult C, Fried LP, Boult L, Wu AW. Clinical practice guidelines and quality of care for older patients with multiple comorbid diseases. JAMA. 2005;294:716-24.

11. Hughes L, McMurdo MET, Guthrie B. Guidelines for people not for diseases: the challenges of applying UK clinical guidelines to people with multimorbidity. Age Ageing. 2013;42(1):62-9.

12. Halpina DMG. Lessons from the major studies in COPD: problems and pitfalls in translating research evidence into practice. Prim Care Respir J. 2010;19:170

13. Carey MP, Senn TE, Vanable PA, Coury-Doniger P, Urban MA. Do STD clinic patients who consent to sexual health research differ from those who decline? Findings from a randomized controlled trial with implications for the generalization of research results. Sex Transm Dis. 2008;35(1):73-7

14. Fortin M, Dionne J, Pinho G, Gignac J, Almirall J, Lapointe L. Randomized controlled trials: do they have external validity for patients with multiple comorbidities? Ann Fam Med. 2006;4(2):104-8.

15. Jones R, Jones R, McCowan C, Montgomery A, Fahey T. The external validity of published randomized controlled trials in primary care. BMC Fam Pract. 2009;10(1):5

16. He J, Guthrie B, Morales DR. A systematic review of the applicability of clinical trial evidence to real-world patients. Prospero. 2016: CRD42016042282 Available from http://www.crd.york.ac.uk/PROSPERO/ display record.asp?ID=CRD42016042282

17. Miró O, Gil V, Muller C, Mebazaa A, Bueno H, Martin-Sanchez FJ, et al. How does a clinical trial fit into the real world? The RELAX-AHF study population into the EAHFE registry. Clin Res Cardiol. 2015;104(10):850-60.

18. Aaltonen KJ, Ylikyla S, Tuulikki Joensuu J, Isomaki P, Pirila L, Kauppi M, et al. Efficacy and effectiveness of tumour necrosis factor inhibitors in the treatment of rheumatoid arthritis in randomized controlled trials and routine clinical practice. Rheumatology (Oxford). 2017;56(5):725-35.

19. Vashisht P, Sayles H, Cannella AC, Mikuls TR, Michaud K. Generalizability of patients with rheumatoid arthritis in biologic agent clinical trials. Arthritis Care Res (Hoboken). 2016;68(10):1478-88.

20. Radner H, Dixon W, Hyrich K, Askling J. Consistency and utility of data items across European rheumatoid arthritis clinical cohorts and registers. Arthritis Care Res. 2015;67(9):1219-29.

21. Zhang S, Liang F, Li W, Tannock IF. Comparison of eligibility criteria between protocols, registries, and publications of cancer clinical trials. J Nat Cancer Inst. 2016;108(11):djw129.
22. Thake M, Lowry A. A systematic review of trends in the selective exclusion of older participant from randomised clinical trials. Arch Gerontol Geriatr. 2017;72:99-102.

23. Broekhuizen K, Pothof A, De Craen AJM, Mooijaart SP. Characteristics of randomized controlled trials designed for elderly: a systematic review. PLoS One. 2015;10(5):e0126709.

24. Du Vaure CB, Dechartres A, Battin C, Ravaud P, Boutron I. Exclusion of patients with concomitant chronic conditions in ongoing randomised controlled trials targeting 10 common chronic conditions and registered at ClinicalTrials.gov: a systematic review of registration details. BMJ Open. 2016; 6(9):e012265.

25. Schmidt AF, Groenwold RHH, Van Delden JJM, Van Der Does Y, Klungel OH, Roes KCB, et al. Justification of exclusion criteria was underreported in a review of cardiovascular trials. J Clin Epidemiol. 2014;67(6):635-44.

26. Cruz-Jentoft AJ, Carpena-Ruiz M, Montero-Errasquín B, Sánchez-Castellano C, Sánchez-García E. Exclusion of older adults from ongoing clinical trials about type 2 diabetes mellitus. J Am Geriatr Soc. 2013;61(5):734-8.

27. Oude Rengerink K, Kalkman S, Collier S, Ciaglia A, Worsley SD, Lightbourne $A$, et al. Series: pragmatic trials and real world evidence: paper 3. Patient selection challenges and consequences. J Clin Epidemiol. 2017:89:173-80.

28. Guthrie B, Thompson A, Dumbreck S, Flynn A, Alderson P, Nairn M, et al. Better guidelines for better care: accounting for multimorbidity in clinical guidelines (NIHR HS\&DR 11/2003/27). Health Serv Deliv Res. 2017;5:16.

29. O'Hare AM, Hotchkiss JR, Kurella Tamura M, Larson EB, Hemmelgarn BR Batten $A$, et al. Interpreting treatment effects from clinical trials in the context of real-world risk information: end-stage renal disease prevention in older adults. JAMA Intern Med. 2014;174(3):391-7.

30. Bourgeois FT, Shannon MW, Valim C, Mandl KD. Adverse drug events in the outpatient setting: an 11-year national analysis. Pharmacoepidemiol Drug Saf. 2010;19(9):901-10.

31. Li L, Geraghty OC, Mehta Z, Rothwell PM. Age-specific risks, severity, time course, and outcome of bleeding on long-term antiplatelet treatment after vascular events: a population-based cohort study. Lancet. 2017;390(10093): 490-9.

32. Atkins D, Chang SM, Gartlehner G, Buckley DI, Whitlock EP, Berliner E, et al. Assessing applicability when comparing medical interventions: AHRQ and the effective health care program. J Clin Epidemiol. 2011:64(11):1198-207.

33. Guyatt GH, Oxman AD, Kunz R, Woodcock J, Brozek J, Helfand M, et al. GRADE guidelines: 8. Rating the quality of evidence-indirectness. J Clin Epidemiol. 2011;64(12):1303-10.

34. Ahmad N, Boutron I, Dechartres A, Durieux P, Ravaud P. Applicability and generalisability of the results of systematic reviews to public health practice and policy: a systematic review. Trials. 2010;11(1):1-9.

35. Beaver JA, Ison G, Pazdur R. Reevaluating Eligibility Criteria — Balancing Patient Protection and Participation in Oncology Trials. N Engl J Med. 2017; 376:1504-5.

36. Guthrie B, Makubate B, Hernandez-Santiago V, Dreischulte T. The rising tide of polypharmacy and drug-drug interactions: population database analysis 1995-2010. BMC Med. 2015;13:74

37. Guthrie B, Boyd CM. Clinical guidelines in the context of aging and multimorbidity. Public Policy Aging Rep. 2018;28(4):143-9.

\section{Publisher's Note}

Springer Nature remains neutral with regard to jurisdictional claims in published maps and institutional affiliations.

Ready to submit your research? Choose BMC and benefit from:

- fast, convenient online submission

- thorough peer review by experienced researchers in your field

- rapid publication on acceptance

- support for research data, including large and complex data types

- gold Open Access which fosters wider collaboration and increased citations

- maximum visibility for your research: over $100 \mathrm{M}$ website views per year

At $\mathrm{BMC}$, research is always in progress.

Learn more biomedcentral.com/submission 